\title{
Torres costeras durante el siglo XVI. Estrategias territoriales y técnicas constructivas en el frente marítimo levantino del Reino de Aragón y Virreinato de Nápoles
} José Ramón Ruiz-Checa ${ }^{\text {a }}$, Valentina Cristinia ${ }^{\text {, Valentina Russo }}{ }^{\text {b }}$

${ }^{a}$ Universitat Politècnica de València, Spain, joruiche@csa.upv.es, vacri@cpa.upv.es, ${ }^{b}$ Università degli Studi di Napoli Federico II, Napoli, Italia, valentina.russo@unina.it

\begin{abstract}
The historical strong relationship between the eastern coast of Spain and southern part of Italian Peninsula has been a common issue over different civilizations from Phoenicians, Greeks and Romans up to the most recent modern kingdoms. Considering this set of connections based on trade routes and territorial bilateral interests, the western shores of Mare Nostrum have been key-points of a dense network of military and economical strategies. The dynamics of extensive territorial control have left interesting examples of costal watchtowers both in the Sorrento Peninsula, near Naples (Italy) and in the East coast of Valencia (Spain). The process of towers construction begins at early stages of Middle Age and, from the 16th century it runs up into a systemic control of the coastline, thanks to the reuse of obsolete fortified elements and new building-site procedures. In the paper, the authors aim at highlighting the territorial relationships among a number of towers built during the 16th century, as well as they propose a comparison of their construction techniques in order to gain an historical comparative and a contextual understanding of these fortified systems.
\end{abstract}

Keywords: construction techniques, landscape, preservation.

\section{Introducción}

A lo largo del siglo XVI distintos enclaves de la costa mediterránea organizaron una importante vertebración operativa de sus territorios orientada a responder de manera satisfactoria a la amenaza marítima de la piratería.

A pesar de configurar realidades alejadas entre sí, el frente marítimo del Reino de Aragón, concretamente la zona costera del Reino de Valencia y el frente costero del Virreinato de Nápoles, representado en este caso por el peculiar enclave de la Península SorrentinaAmalfitana, constituyen dos interesantes realidades geográficas afines convergiendo en un mismo control político y, por otro lado, en un escenario de incursiones y pillajes (González Ruiz 2012). Los objetivos del estudio radican por lo tanto en una confrontación de soluciones estratégicas y constructivas, analizando divergencias y convergencias de las propuestas arquitectónicas vinculadas a torres costeras, localizadas o documentadas en estas dos áreas de estudio.

\section{Fortificaciones costeras en el siglo XVI.} Ubicación y relación con el paisaje

La costa mediterránea es, históricamente, un espacio ambivalente: por un lado, ha permitido un escenario idóneo para establecer contactos comerciales, fomentado la actividad económica, la pesca y el intercambio cultural y social de las poblaciones ribereñas. Al mismo tiempo, las particularidades de este perfil marítimo, a lo 
largo de los siglos, se centran, sin duda en una franja territorial peligrosa y difícil de controlar, provocando, en muchos episodios históricos, el debilitamiento de políticas o la economía de muchas monarquías europeas. Dentro de las medidas dirigidas a evitar razzias y amenazas de ataques marítimos, la construcción de torres defensivas es una estrategia decisiva que ve en el siglo XVI un momento crucial para la definición y desarrollo de un frente, lo más continuo posible, a partir de enclaves fortificados por el Mediterráneo (Braudel 1976). Según esta lógica, las costas ibéricas desde Gibraltar, la costa de Granada, Murcia, los lindes valencianos y catalanes viven en este siglo importantes obras de fortificación (Cámara 1998-2005).

Fuera de los reinos peninsulares, Italia es otro buen ejemplo de estas soluciones constructivas, con los casos de Nápoles, Sicilia y Cerdeña, entre otros. Más allá del avance de los usos de artillería, estas torres vigías que salpican el Mediterráneo cuentan con influencia del incipiente Humanismo que despierta en toda Europa, aprovechando los recursos de la naturaleza que ofrecen las costas del Mare Nostrum, y por otro lado, captando y combinando los recursos naturales con las tecnologías constructivas. Se genera así, un auténtico litoral construido y estructurado con base defensiva. Pero, sin duda, el objetivo de defensa será tanto bélico como de protección de núcleos de población situados próximos a la costa, calas, islas, penínsulas o puertos naturales.

\subsection{Estrategias territoriales en el frente marítimo del Reino de Aragón}

A lo largo del siglo XVI, la amenaza de los piratas turco-berberiscos es evidente $\mathrm{y}$ alarmantemente creciente. Este hecho potencia y justifica en parte, el aumento de recursos militares en tierra firme, para poder garantizar la precaria defensa del litoral.

En el caso del Reino de Valencia, como cara expuesta del Reino de Aragón, se configura durante este siglo una evolucionada e interesante red de torres y castillos que protegen una serie de enclaves estratégicos situados tanto en la línea costera como en el interior del territorio valenciano. La eficacia del sistema defensivo radicará, realmente, en la continua negociación entre el rey, representado por el virrey de Valencia, y las instituciones como Juntas Estamentales, Generalitat y/o Municipalidades. A partir de 1525, y en estrecha relación con la vigilancia sobre la población morisca, sometida a duros decretos de conversión forzosa, se organizan pequeñas compañías de infantería y caballería, con la misión de patrullar por los caminos y las zonas costeras (Belenguer 2001). Poco a poco, esas compañías, financiadas y controladas por las instituciones regionales, se consolidan como cuerpos armados estables, y hacia 1550 se les dota de recursos, aumentando así su movilidad y autonomía.

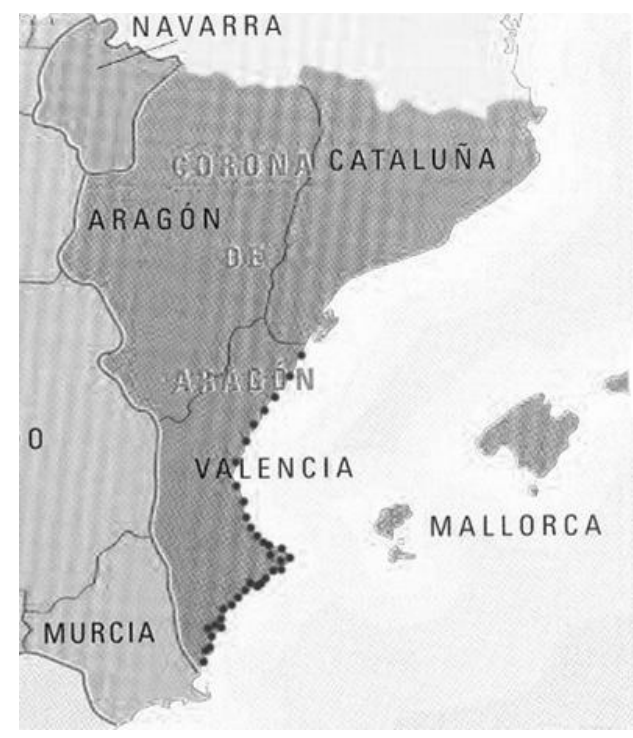

Fig.1- Emplazamiento de las torres costeras localizadas en los límites territoriales del Antiguo Reino de Valencia (V. Cristini-JR Ruiz).

\subsection{La elección de implantación en el Virreinato de Nápoles}

Las incursiones marítimas turco-berberiscas en la costa de Sorrento y Massa Lubrense, llevadas a cabo en junio de 1558, provocan la planificación de un sistema fortificado en el territorio del Virreinato de Nápoles (Pasanisi 1926, Santoro 1982, Mafrici 1989, Russo 2009). A pesar de no disponer de una descripción gráfica de los sistemas de fortificación, se puede 
establecer que la elección de ubicaciones, llevada a cabo por el virrey Perafan de Ribera, duque de Alcalá y de su cuerpo de ingenieros, responde a un claro criterio de control contra las incursiones. La definición racional en el posicionamiento e implantación de las torres de avistamiento y defensa queda intrínsecamente unida a la definición arquitectónica. En este sentido, hay que resaltar dos factores clave, por un lado, la extremada complejidad territorial de los casi $2000 \mathrm{kms}$ de línea costera, y por otro, en el breve lapso de tiempo en el que se lleva a cabo la iniciativa. Se trata, no solo, de nuevas construcciones, sino también, de la restauración de preexistencias tanto en la línea costera como en el interior de la zona meridional de la península (Campania, Basilicata, Puglia y Calabria) (Faglia 1970, Id. 1975; Id. 1984).

En este contexto, la geomorfología del promontorio entre los golfos de Nápoles y de Salerno, históricamente, ha convertido a este territorio en una barrera para el tráfico marítimo y las conexiones entre los diferentes núcleos urbanos de Nápoles, Amalfi y Salerno. Por otro lado, también, este enclave se convierte en un puente en los ámbitos sociales y económicos. Es decir, el principal tipo de interconexión practicado es "transversal", caracterizado por tránsitos desde un punto costero hacia el interior. Este tipo de relación territorial está muy alejada del modelo de interconexión "longitudinal", que toma como directriz la costa y que se implanta a partir del siglo XIX.

El sistema fortificado analizado, además de seguir una lógica militar o constructiva, potencia unos lazos creados entorno a aspectos sociales y/o económicos, todo ello se puede evidenciar siguiendo una lectura estratigráfica del paisaje a través de condicionantes naturales y acciones antrópicas. La vulnerabilidad del territorio peninsular se detecta, además, en algunas referencias medievales de época angioina. En 1277 Carlos I ordena la defensa de la costa, y tal y como indica Filangieri, en esta franja de territorio aparecen algunas preexistencias como la Torre de Minerva, en Punta Campanella o la Torre de Capo Corbo (Filangieri 1910, p. 142 y p. 241). Otra posible preexistencia se refiere a un promontorio fortificado recogido en las cartografías aragonesas de Giovanni Pontano (La Greca, Valerio 2008).

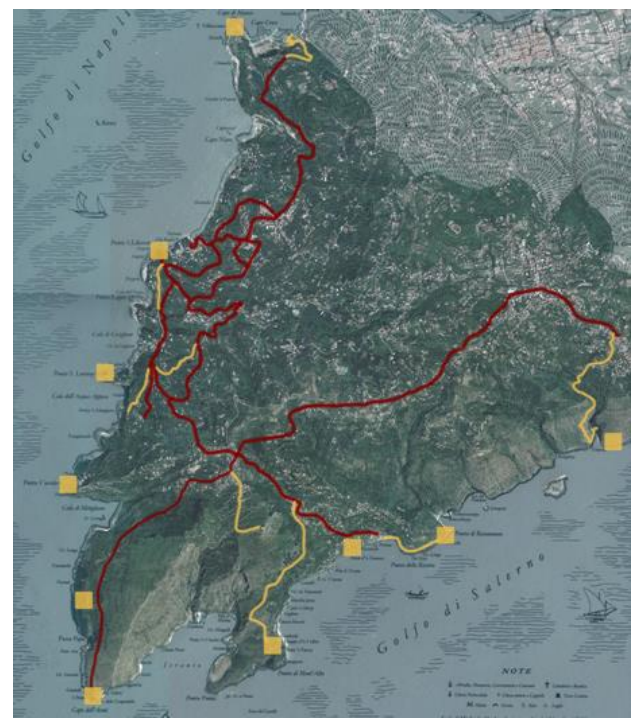

Fig. 2- Emplazamiento de las diez torres virreales en la Peninsula Sorrentina-Amalfitana en relación con el sistema histórico de vías de comunicación (elab. I. Barone Ms Thesis).

En 1567 son contratados los trabajos de torreggiamento de la Península sorrentina a los constructores Cafaro Pignaloso y Ettore Cafaro (Santoro 2000). En 1570 la mayor parte de las torres serán entregadas a la Corona para: controlar los movimientos marítimos y gestionar el acceso al gran número calas con que cuenta esta accidentada costa, contando para ello con dos sistemas superpuestos, por un lado, torres costeras erigidas a lo largo del frente litoral, y por el otro, un sistema de casas fuerte en zonas internas de la península.

\section{Construcciones históricas y reconstrucción de las torres costeras un estudio comparado}

Queda constancia que en 1563 se propone remodelar las torres próximas a Valencia para adecuarlas a los nuevos métodos de defensa (Braudel, p. 906, cit. in Russo 2009, p. 124). 
En el mismo año se inician las obras del sistema de fortificaciones de la costa meridional italiana gracias al planteamiento propuesto por el virrey Perafan de Ribera. Estos hechos arrojan más pistas a la hora de entender cómo las costas del Mediterráneo cuentan desde época remota con sistemas de defensa, tal y como demuestran los restos arqueológicos de emplazamientos griegos, romanos o islámicos. En muchos casos, hay que reconocer que estas soluciones arcaicas cuentan con sistemas estratégicos bastante rudimentarios, con un modelo que se apoya en la red de vigilancia terrestre, cubriendo limitados tramos de costa. También, a veces, es frecuente encontrar datos de sistemas de vigilancia fija pero $\sin$ estructuras defensivas, que cuentan simplemente con guardias de ronda o galeras. Considerando este marco histórico, el sistema defensivo del litoral valenciano, así como las estructuras de las costas meridionales de la península itálica, viven un momento de cambio a partir de la segunda mitad del siglo XVI, cuando se organiza definitivamente una estructura organizada y jerarquizada de torres de defensa, cuyos rasgos principales se definen a finales del siglo, tras continuas revisiones y puesta a punto por los ingenieros reales. Varios experto italianos, como Giorgio Palearo, Pedro Velasco o Giovanni Battista Antonelli entregan numerosos documentos e informes al virrey, especialmente en el entorno de Valencia, Alicante, Cullera y Dénia (Boira Maiques 2007). Se trata de documentos importantes ya que permiten averiguar como en todo el litoral, en 1563, existen 46 torres recién ejecutadas, 14 en proyecto y también una serie no bien definida de actuaciones menores en curso. A estos se suman los informes del virrey de Valencia, Vespasiano Gonzaga y Colonna (1575) y del veedor real Juan de Acuña (1585), tratándose de importantes documentos que atestiguan el fervor defensivo que se vive en la costa. La novedad que se impone en el diseño propuesto por estos ingenieros en ambos contextos de estudio es la de superar el sistema medieval de alerta y prevención y transformarlo en una red potente de respuesta a los ataques, gracias a un sistema fortificado con artillería.

\subsection{Las técnicas constructivas en el contexto del frente litoral del Reino de Aragón}

El litoral del Antiguo Reino de Valencia (con aproximadamente $80 \mathrm{kms}$ de largo) cuenta con un perfil más ondulado en su mitad norte (con playas de arena, deltas, humedales), bastante más accidentado y complejo es el sur, donde la costa es perfilada con acantilados y presenta una configuración más abrupta y difícil. Este hecho geográfico influye en la concentración de torres vigías justo en las zonas meridionales, territorialmente más vulnerables.

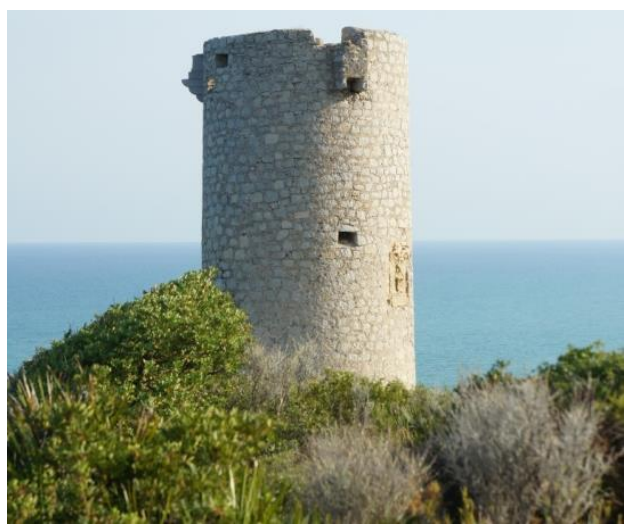

Fig. 3- Sierra D'Irta (Peñiscola). Torre Almadum ejemplo de torre cilíndrica (V. Cristini-JR Ruiz).

Como en el caso napolitano, la presencia de recursos constructivos, ríos y canteras, agiliza la edificación de un sistema defensivo.

Para comprender la realidad y la materialidad constructiva de estas torres existen interesantes estudios llevados a cabo en los últimos años, aunque todos ellos con una perspectiva más geográfica e histórica que arquitectónica. Los más destacados son posiblemente el mapeo de Boira Maiques (2007) junto con los estudios de Cámara Muñoz (2005). Gracias a estas contribuciones hasta la fecha se han documentados 54 torres, 12 de ellas sólo a nivel de emplazamiento geográfico y datos documentales genéricos, no quedando trazas constructivas evidentes.

Una veintena de ejemplos cuentan con un cuerpo prismático (más resistente que los volúmenes cilíndricos o cónicos), ideal para defender los 
puertos de las villas o puntos estratégicos como salinas o desembocaduras de ríos.

Algunas a destacar son las Torres de Grao de Castellón, Borriana, Almenara, Sagunto, Gandía, de la Sal, de Capicorb, del Pinet, de Sól de Riu o Torre Nova de les Salines (Boira Maiques 2007). Normalmente, estos elementos austeros y robustos presentan una altura de $10 / 13 \mathrm{~m}$, una planta de $25-32 \mathrm{~m}$ de perímetro cuadrangular. Usualmente, la estructura es de calicanto o mampostería y se caracteriza por la presencia de una puerta con sillares a ras del suelo (para abrigar atajadores y sus caballos con los que recorrían el litoral).

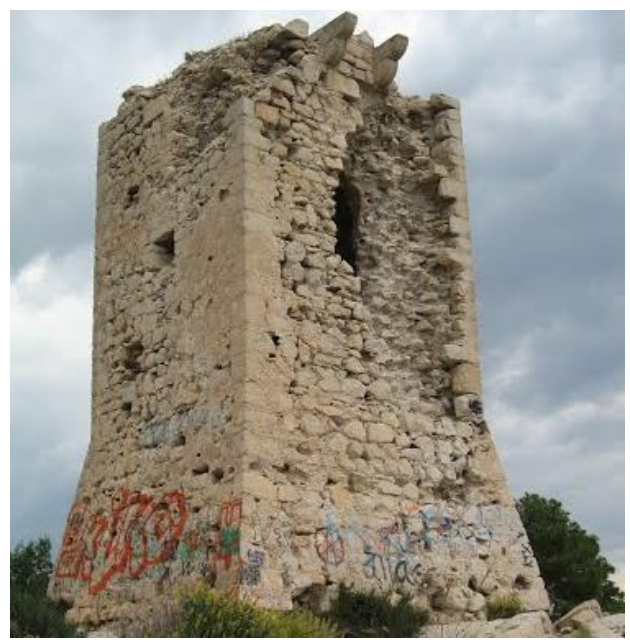

Fig. 4- Torre de L'Aguiló (Villajoyosa). Detalle previo a la intervención: muros a saco, con mampuestos y con sillería en las esquinas ( $\mathrm{J}$. Ramos).

En sección, es común, la presencia de un espacio tripartido, con planta baja (a veces con abrevaderos para caballos, aljibes o pozos) más dos pisos y terrazas. Se trata de espacios cubiertos por bóvedas de cañón y comunicados entre sí por escalerillas de madera, retiradas en caso de asedio. También es frecuente la presencia de remates con sillería, como guirnaldas, parapetos, merlones o matacanes, también en algunos casos, taludes de refuerzo, cuyas trazas todavía no siempre se conservan o están bien documentadas. Las nueve torres que hoy día todavía se conservan, presentan rasgos constructivos marcados por un cuerpo troncocónico, siendo caracterizadas por plantas circulares de aproximadamente $11-12 \mathrm{~m}$ de diámetro que se estrecha hasta los $6-7 \mathrm{~m}$ en su remate. Las que están situadas en lugares de difícil acceso cuentan con estructuras más simples, de altura más limitada, que presentan una base maciza sobre la que se levanta un único habitáculo, puerta de acceso elevada y una terraza, separada por una resistente bóveda de cañón. Las principales torres a destacar de esta tipología son la Torre de la Gola de Xúquer, de les Caletes de Benidorm, de les caletes de Santa Pola, de cap de Moraira, de la Foradada, de cap Cerver o cap Roig (Boira Maiques 2007).

Las restantes torres presentan un cuerpo cilíndrico, caracterizado por planta circular de $11-12 \mathrm{~m}$ de diámetro, usualmente con base maciza, marcada por un talud de protección hasta un tercio de su altura, comprendida entre $10-13 \mathrm{~m}$. Emulando las características de las torres con cuerpo troncocónico estas tipologías también poseen un único piso, puerta elevada, bóveda de cañón con apertura para acceder a la terraza. Destaca en este sistema la Torre del Giralei o la de Campello (Boira Maiques 2007). Sin duda, las intervenciones realizadas en las torres en las últimas décadas dificultan la lectura estratigráfica o constructiva, debido a la presencia de enlucidos o rejuntados sistémicos de fábricas históricas. No obstante, es posible reconocer algunos datos de la materialidad de estos edificios, especialmente en casos no intervenidos, o gracias a fotos de patologías de degradación sufrida por algunos elementos. La sección constructiva en varios casos manifiesta la presencia de muros a saco, realizados con bastos morteros de cal, con árido irregular y cocción rápida, con presencia de caliches con partes mal cocidas, tal y como se puede apreciar en algunos ejemplos de la costa napolitana.

\subsection{Materiales de construcción en el contexto de la Península sorrentino-amalfitana}

La relativa facilidad de disponibilidad de agua dulce en la Península Sorrentina se debe al gran número de torrentes que surcan su topografía. Se trata de terrenos calcareníticos con una 
estructura estratificada óptima para su utilización en construcción. El caso de estudio contempla el análisis de nueve enclaves más la Torre Baccola, la cual prácticamente ha desaparecido. En todos ellos, se pueden establecer dos sistemas de medidas, uno con base cuadrada, con uno de los lados entre 10$13 \mathrm{~m}$ y otro sistema, como el caso de la Torre San Lorenzo y la Torre de Recommone, cuyos lados son algo más reducidos que los edificios anteriores, de planta de $8 \mathrm{~m} \quad(32$ palmos napolitanos). Justo en estos últimos ejemplos, es donde se pueden establecer conexiones visuales entre ellos, pudiendo considerarse torres de guarda, las cuales fueron proyectadas cómo espacios mínimos, sin servicios, para la presencia puntual, inferior a las $24 \mathrm{~h}$, de un torrero.

\section{La Torre de San Pietro en Crapolla (Delizia} 2014; Santaniello 2014) posee una doble función defensiva y vigilancia sobre el frente meridional de la Península. Esta torre puede considerarse uno de los mejor ejemplos conservados, y cuyo interés en el estudio radica en la posibilidad de entender perfectamente el sistema constructivo empleado y el rol que esta arquitectura adquiere en el marco de la iniciativa virreinal. Se trata de una construcción emplazada en una profunda cala homónima, la cual estaba conectada a través de un escarpado sendero con el núcleo de la población. $\mathrm{Su}$ visual marítima domina perfectamente el archipiélago de Li Galli. Además de la defensa contra el corso, la torre cumple otra función, como es, la de protección de la abadía benedictina de San Pietro, situada a escasos metros de la cala, lugar de suministro de agua potable procedente del arroyo Iarito. Las condiciones ambientales, por lo tanto, tuvieron que empujar en la selección del sitio, en correspondencia visual con las torres Recommone y Montalto hacia el oeste y la torre Castelluccia en la isleta de Gallo Lungo.

La Torre de San Pietro posee un volumen tronco piramidal, siguiendo el talud progresivo de los alzados laterales con unas zarpas de aproximadamente $8^{\circ}$ de inclinación. Esta opción constructiva consolida estructuralmente el volumen y cumple con criterios pirobalísticos, ya que la torre está expuesta a los ataques desde el mar. Durante la construcción de la torre se suministra el material pétreo, gracias a las excavaciones necesarias para configurar la cisterna. El nivel superior, correspondiente con el nivel de la ladera, constituyendo el espacio habitable de la construcción, en la cual el torrero puede alojarse con las mínimas comodidades. Por otra parte, desde este mismo espacio, el guarda puede realizar las labores de vigilancia del territorio gracias a diferentes vanos abiertos estratégicamente en tres de sus frentes. En dicha composición interna, podemos encontrar una chimenea, una letrina, espacios de almacenaje así como elementos de iluminación. Incluso el agua de cubierta es aprovechada y canalizada a través de conductos de piezas de cerámica (cotto), de los cuales únicamente tenemos constancia gracias a su impronta sobre la fábrica. Desde el nivel de acceso se asciende al plano de cubierta gracias a una escala, conservada hoy día en malas condiciones. En este nivel aparece el puesto de guardia y el espacio para municiones. El perímetro de dicha cubierta queda protegido por un parapeto con merlones en contraescarpa a modo de tronera.

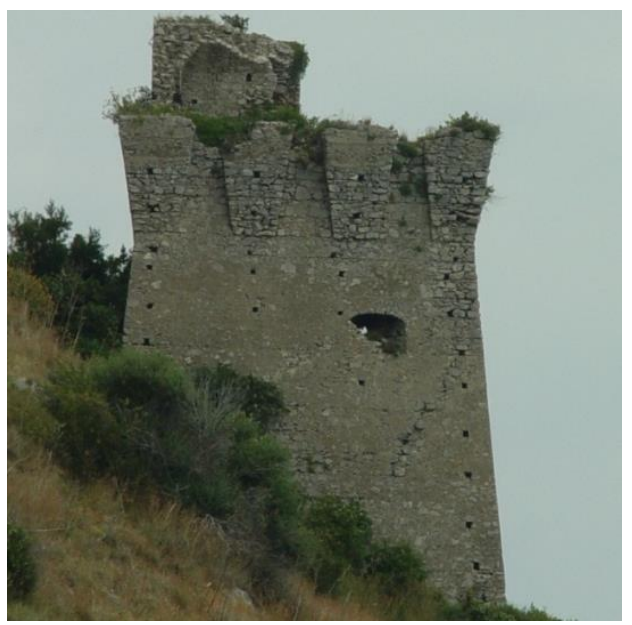

Fig.5- Massa Lubrense (Napoles). Alzado oeste de Torre di San Pietro (V. Russo).

Desde el punto de vista funcional, en la menos imponente Torre de Recommone no aparecen huellas de elementos destacados o recursos de uso militar o de vigilancia, especialmente en el nivel de cubierta. No obstante, estos dos edificios, gracias a su grado de conservación y a 
su fácil accesibilidad, permiten apreciar perfectamente las esmeradas labores llevadas a cabo en la obra del siglo XVI, con importantes detalles constructivos y datos de la sección de las fábricas. Los dos elementos, además, presentan paramentos a base de muros a saco, con abundante mortero y mampuestos (piezas regulares con aparejo horizontal), que pueden ser colocados en obra por un solo operario. Las fábricas testimonian un riguroso orden en su disposición y ejecución, como por ejemplo el empleo de dimensiones antrópicas (palmo napolitano) en las disposiciones de las hiladas. Se trata de franjas horizontales - que coinciden con jornales (cantieri) de aproximadamente 70 $\mathrm{cm}$ de alto - que sirven como referencia para el trazado y replanteo de elementos específicos de la torre como es el arranque de la bóveda, las alturas de los vanos, la dimensión de las almenas y merlones. Estos jornales aumentan en altura en correspondencia con los puntos de descarga de las bóvedas centrales y se reducen en las zonas próximas a las troneras, tratándose de puntos críticos a ejecutar en la fábrica (Santaniello 2014).

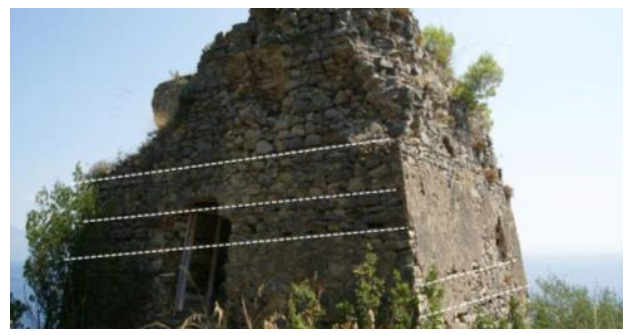

Fig. 6- Massa Lubrense (Napoles). Torre de Recommone. Detalle (líneas blanca) de las fajas de jornales que se pueden apreciar en los alzados (V. Russo).

En el caso de la Torre de San Pietro, además, los mechinales quedan alineados verticalmente (aproximadamente cada dos jornales) y horizontalmente (respetando las cuatro fajas que corresponden a la mediana de las barbacanas de la contraescarpa de coronación). Durante la toma de muestras y pruebas petrográficas (abril 2013), se ha constatado la presencia de caliches y puzolanas, como aditivos, mejorando el comportamiento frente al agua de algunos paños expuestos a la intemperie como son la zona de cubierta y las zonas de coronación (Gabrielli 2014, p. 144 у p. 147).

\section{Conclusiones}

En el presente trabajo se ha recurrido a un proceso de investigación comparada en el ámbito mediterráneo, considerando la macroescala del paisaje o enclaves territoriales y la micro-escala del detalle y técnicas estratigráficas vinculadas al palimpsesto construido. En el caso de España, la presencia de torres vigías en la línea costera del Reino de Valencia encuentra un obstáculo en las dificultades políticasestratégicas del frente marítimo, concretamente en la negativa de las élites regionales, parapetadas en sus instituciones tradicionales, a integrarse en una organización militar unificada, dirigida por la Corona y su estructura de mando (AA.VV., 1973). A nivel constructivo y arquitectónico las torres litorales valencianas, así como las torres analizadas en el contexto meridional italiano, han sido estudiadas $\mathrm{y}$ definidas con exhaustividad desde una lógica geográfica e histórica, pero de forma bastante fragmentaria desde el punto de vista constructivo y arquitectónico. La variedad tipológica, de hecho, con variaciones de plantas y alzados (se clasifican ejemplos de cuerpos prismáticos, troncocónicos, troncopiramidales, cilíndricos) permite apostar por una lectura de estos edificios más articulada en el tiempo, con la posibilidad de incluir reutilización de preexistencias previas a las estructuras del siglo XVI. A la vez, surgen posibles conexiones a trazar entre los dos contextos, italiano e ibérico, como son las lógicas sistémicas empleadas, vinculadas a las nuevas teorías humanistas, capaces de adaptarse al territorio, antropizarlo de forma rigurosa y organizada, según las pautas que el Renacimiento impone en las prácticas constructivas. También es interesante buscar afinidad en los recursos constructivos de ambos sistemas fortificados. Se marca de hecho el empleo de piedras calizas de ágil y rápida puesta en obra, con muros a saco, rudos morteros de cal, sillares regulares para esquinas o vanos. También existe una cierta homogeneidad de medidas antropométricas, al margen de ligeras variaciones geométricas. La modulación antrópica de los espacios se extiende también en 
los alzados (10-13m de altura) y en planta $(30 \mathrm{~m}$ aprox. de perímetro).

La investigación por parte de los autores españoles ha sido llevada a cabo en el marco del proyecto“ Trazabilidad histórica y perspectivas para los materiales sostenibles vinculados a la tradición constructiva de La Comunidad Valenciana, Ayudas GV 2014/014".

\section{Referencias}

AA.VV. (1973), VIII Congreso de Historia de la Corona de Aragón. La Corona de Aragón en el siglo XVI. Caja de Ahorro y Monte de Piedad de Valencia Ed. pp. 54-65.

AA.VV. Sistema defensivo de Felipe II, Rutas de torres vigía de la Costa de Alicante, GVA Ed., Alicant (visor web http://torresvigia.icv.gva.es/)

Belenguer E. (2001), La Corona de Aragón en la monarquía hispánica: del apogeo del siglo XV a la crisis del XVIII, Península Ed. Barcelona.

Boira Maiques J.V. (2007), Las torres del litoral valenciano, Consellería de Infr.y Trans. Ed. Valencia.

Braudel F. (1976), El Mediterráneo y el mundo mediterráneo en la época de Felipe II, vol. II, Fondo de Cultura Económica Ed., 1976 (2 ed), México DF. cap. 6.

Cámara Muñoz A. (2005), Los ingenieros militares de la monarquía hispánica en los siglos XVI y XVII, Ministerio de Defensa Ed., Madrid. cap. 5-6.

Cebrián, R. (2014). Caminos junto al mar: Comunitat Valenciana, Ed. Carena, L'Eliana

Delizia F. (2014). St. Peter's Tower at Crapolla as an example of the defensive system of the Sorrentine Peninsula in the viceroyal age in Russo V. (coord.) (2014). Landscape as Architecture. Identity and conservation of Crapolla cultural site. Nardini. Firenze. pp. 123-128.

Faglia V. (1970). Contributo alla conoscenza delle torri costiere in Terra di Bari. Ist. Italiano dei Castelli. Roma.

Faglia V. (1975). Tipologia delle torri costiere nel Regno di Napoli. Istituto Italiano dei Castelli. Roma.

Faglia V. (1984). Tipologia delle torri costiere di avvistamento e segnalazione in Calabria Citra in Calabria Ultra dal XII . secolo. Istituto Italiano dei Castelli. Roma.

Filangieri di Candida R. (1910). Storia di Massa Lubrense. Pierro. Napoli.

Gabrielli R. (2014). Ancient mortars in the archaeological site of Crapolla: laboratory analysis in Russo V. (coord.) (2014). Landscape as Architecture. cit. pp. 137-147.

González Ruiz D. (2012), Breve historia de la Corona de Aragón, Nowtilus Ed., Madrid. pp.55-59.

La Greca F., Valerio V. (2008). Paesaggio antico e medioevale nelle mappe aragonesi di Giovanni Pontano. Le terre del Principato Citra. Edizioni del Centro di promozione culturale per il Cilento. Acciaroli.

Mafrici M. (1989). La difesa delle coste meridionali nei secoli XVI-XVII: tecnici e tecnologie in Placanica A. (coord.). Annali del Centro Studi "A. Genovesi" per la storia economica e sociale. ESI. Napoli. pp. 31-106.

Pasanisi O. (1926). La costruzione generale delle torri marittime ordinata dalla Regia Corte di Napoli nel XVI secolo in Studi di storia napoletana in onore di Michelangelo Schipa. I.T.E.A. Napoli.

Russo F. (2001). Le torri anticorsare vicereali con particolare riferimento a quelle della costa campana. Istituto Italiano dei Castelli - sez. Campania. s.l.

Russo F. (2009). Le torri costiere del Regno di Napoli. La frontiera marittima e le incursioni corsare XVI ed il XIX secolo. ESA. Napoli.

Santaniello E. (2014). The cantieri masonry of St. Peter's Tower. Building techniques and similarities in the peninsular context in Russo V. (coord.) (2014). Landscape as Architecture. cit., pp. 129-136.

Santoro L. (2000). "Torri costiere da Rovigliano a Vietri" in Apollo. XVI. pp. 17-113.

Sarasa Sánchez E., Serrano E (1997), La Corona de Aragón y el Mediterráneo: Siglos XV-XVI, Institución Fernando El Católico Ed., Madrid. pp. 78-95.

Suárez Fernández L., Gallego J.A. (1993), La Crisis de la hegemonía española, siglo XVII, Rialp Ed., San Sebastián. cap. 4. 